\title{
Évolution et détermination de la flore bactérienne d'un lait cru réfrigéré paucimicrobien
}

\section{en fonction du temps}

\author{
par \\ R. Bloquel et L. Veillet-Poncet*
}

\section{INTRODUCTION}

Le but essentiel visé par le refroidissement du lait cru à la ferme est de préserver ses propriétés originelles jusqu'au moment de son traitement.

Cependant la durée de stockage acceptable dépend du degré de contamination du lait et elle est en particulier limitée par la croissance d'organismes psychrotrophes, capables de se multiplier activement aux températures de réfrigération.

Après avoir étudié l'évolution quantitative des flores aérobies, mésophile et psychrotrophe, d'un lait cru réfrigéré peu contaminé, conservé à $4^{\circ} \mathrm{C}$ pendant $4 \mathrm{j}$ [2], nous nous proposons de déterminer si, dans ces conditions, le stockage du lait peut ou non être à l'origine d'une sélection microbienne et, par là même, d'une modification de la nature et de l'équilibre de la microflore de ce lait cru.

\section{METHODOLOGIE}

\section{Isolement de la flore bactérienne dominante}

Les colonies représentatives de la flore bactérienne dominante mésophile et psychrotrophe sont isolées selon les techniques classiques, à partir de quinze échantillons de lait cru réfrigéré à $4^{\circ} \mathrm{C}$ aux stades $\mathbf{J}(20 \mathrm{~h})$ et $\mathbf{J}+4(96 \mathrm{~h})$. Une fois la pureté des clones vérifiée,

* Laboratoire de Microbiologie Alimentaire E.N.S.A.I.A.-I.N.P.L., 32, rue SainteCatherine - 54000 Nancy (France). 
ces derniers, cultivés sur milieu nutritif gélosé incliné, sont conservés à $4^{\circ} \mathrm{C}$ jusqu'à leur identification [1].

\section{Identification des clones}

A partir d'une culture de 24 h sur milieu gélosé nutritif, les tests suivants sont effectués : la coloration de Gram qui permet aussi l'observation de la morphologie cellulaire, la recherche de la catalase, de l'oxydase [40], de la cytochrome-oxydase [21], de la production ou non de pigments diffusibles ou non.

Les identifications sont effectuées en se référant au "Bergey's Manual » de 1974 [16] et en utilisant conjointement les monographies proposées par différents auteurs et nos propres schémas de détermination [1].

\subsection{Les bactéRIes Gram positives}

- Micrococcaceae

L'étude du type respiratoire, le mode de dégradation du glucose $[3,2]$ et l'étude de l'arginine décarboxylase [57] nous permettent de différencier les genres: Micrococcus et Staphylococcus.

Les sous-groupes des deux genres sont respectivement identifiés selon les critères retenus par Baird-Parker [4].

\section{- Bacillaceae}

La forme et la position de la spore colorée selon la méthode de Wirtz permet de distinguer trois groupes. Le type respiratoire est défini sur milieu viande levure.

Les espèces du genre Bacillus sont identifiées en faisant référence aux travaux de Knight et Proom [38], Wolf et Barker [86], Lemille [43] et de Barjac et al. [17].

\subsection{Les bactéries Gram négatives}

L'identification est essentiellement fondée sur la morphologie cellulaire, la réponse au test de l'oxydase, l'étude du système ciliaire, la présence de pigments ; le mode de dégradation du glucose sur milieu de Hugh et Leifson [30] permet de distinguer les bactéries de type fermentant, oxydant inerte et alcalinisant.

En dehors de ces critères, notre schéma d'identification tient compte des travaux de Shewan [69], Hendrie [28], Pickett [58], King [35] et Blazevic [8] en ce domaine.

\subsubsection{LES BACTÉRIES FERMENTANTES}

\section{- Vibrionaceae}

Le genre Aeromonas isolé est identifié en faisant référence aux travaux de Schubert [62], Veillet-Poncet [82] et Popoff [59]. 


\section{- Enterobacteriaceae}

Les espèces sont différenciées selon Edwards et Ewing [20] et Le Minor [45]. L'aspect du milieu de Kliger, la recherche de la $\beta$ galactosidase [44], la réponse aux tests I.M.V.I.C., l'étude des fermentations sucrées, de la mobilité, l'hydrolyse de la gélatine et la recherche d'une désoxyribonucléase [7] permettent d'identifier les souches isolées.

\subsubsection{LES BACTÉRIES OXYDANTES}

- Les bactéries fluorescentes

La production de pyoverdine, pigment fluorescent vert, diffusible, est recherchée sur milieu B de King [83, 72].

L'identification, fondée sur les travaux de Shewan et al. [66], Stanier [70], Sutter [72], tient compte de la nomenclature de la $8^{\mathrm{e}}$ éd. du manuel de Bergey [16] et repose aussi sur nos propres investigations [1].

- Les bactéries non fluorescentes

L'absence de production de pyoverdine est vérifiée selon les recommandations de Veron [83.] et Sutter [72]. Les caractères retenus pour l'identification sont ceux préconisés par le manuel de Bergey [16], par Seleen [66], Stanier [70] et Sutter [72].

\subsubsection{LES BACTÉRIES INERTES}

Les bactéries inertes sur milieu de Hugh et Leifson glucosé sont identifiées en fonction des caractéristiques suivantes : croissance ou non à $41^{\circ} \mathrm{C}$, dénitrification ou non, action sur le lait tournesolé, présence ou absence d'amylase, de gélatinase, de lécithinase d'argininedihydrolase [81] et selon les critères établis par Stanier [70], Gilardi $[23,25,26]$, Juffs [34] et le manuel de Bergey [16].

\subsubsection{LES BACTÉRIES ALCALINISANTES}

Leur identification est fondée sur les caractères retenus pour l'identification des bactéries inertes et sur l'épreuve de sensibilité à la pénicilline G à 2,5 unités. Achromobacter est identifié par référence aux travaux de Buttiaux [13, 15], Baumann [5], Shewan [68], Stenzel [71], Gilardi [23, 24] et Henriksen [29].

\section{RESULTATS ET DISCUSSION}

Le lait cru étudié présente au stade $\mathrm{J}$ une flore aérobie mésophile de $1.10^{4}$ bactéries par $\mathrm{ml}$ et une flore psychrotrophe de $5.10^{2}$ par $\mathrm{ml}$ et au stade $\mathrm{J}+4$, après stockage à $4^{\circ} \mathrm{C}$, ces flores sont respectivement de $1,5.10^{4}$ par $\mathrm{ml}$ et de $4.10^{3}$ par $\mathrm{ml}$ [2]. 


\section{TABLEAU 1}

Répartition des flores bactériennes dominantes en fonction de la coloration de Gram et de la durée de réfrigération du lait à $+4^{\circ} \mathrm{C}$

\begin{tabular}{|c|c|c|c|c|c|}
\hline & \multicolumn{2}{|c|}{$\begin{array}{l}\text { Flore mésophile } \\
\left(30^{\circ} \mathrm{C}-72 \mathrm{~h}\right)\end{array}$} & \multicolumn{2}{|c|}{$\begin{array}{c}\text { Flore psychrotrophe } \\
\left(7^{\circ} \mathrm{C}-10 \mathrm{j}\right)\end{array}$} & \multirow{2}{*}{ Total } \\
\hline & $\begin{array}{c}\text { Stade } \\
(\mathbf{J})\end{array}$ & $\begin{array}{l}\text { Stade } \\
(\mathbf{J}+4)\end{array}$ & $\begin{array}{c}\text { Stade } \\
\text { (J) }\end{array}$ & $\begin{array}{l}\text { Stade } \\
(J+4)\end{array}$ & \\
\hline Bactéries Gram positives & 139 & 85 & 56 & 19 & 299 \\
\hline Bactéries Gram négatives & 30 & 65 & 79 & 143 & 317 \\
\hline Total & 169 & 150 & 135 & 162 & 616 \\
\hline
\end{tabular}

\section{TABLEAU 2}

Répartition des bactéries Gram négatives selon leur réponse au milieu de Hugh et Leifson glucosé et au test de l'oxydase

\begin{tabular}{|c|c|c|c|c|c|c|c|c|c|}
\hline \multirow{2}{*}{$\begin{array}{c}\text { Flore } \\
\text { Gram négative }\end{array}$} & \multicolumn{2}{|c|}{$\begin{array}{c}\text { Bactéries } \\
\text { fermentantes }\end{array}$} & \multicolumn{2}{|c|}{$\begin{array}{l}\text { Bactéries } \\
\text { oxydantes }\end{array}$} & \multicolumn{2}{|c|}{$\begin{array}{l}\text { Bactéries } \\
\text { inertes }\end{array}$} & \multicolumn{2}{|c|}{$\begin{array}{c}\text { Bactéries } \\
\text { alcalinisantes }\end{array}$} & \multirow{2}{*}{ Total } \\
\hline & \multicolumn{2}{|c|}{ oxydase } & \multicolumn{2}{|c|}{ oxydase } & \multicolumn{2}{|c|}{ oxydase } & \multicolumn{2}{|c|}{ oxydase } & \\
\hline Mésophile & 2 & 20 & 47 & 0 & 11 & 0 & 15 & 0 & 95 \\
\hline Psychrotrophe & 2 & 28 & 160 & 0 & 8 & 0 & 24 & 0 & 222 \\
\hline Totale & 4 & 48 & 207 & 0 & 19 & 0 & 39 & 0 & 317 \\
\hline
\end{tabular}

\section{Répartition des souches bactériennes}

Compte tenu de la durée de réfrigération à $4^{\circ} \mathrm{C}$, le tableau 1 rend compte de la répartition des flores bactériennes mésophile ( $30^{\circ} \mathrm{C}$ $72 \mathrm{~h})$ et psychrotrophe $\left(7^{\circ} \mathrm{C}-10 \mathrm{j}\right)$ dominantes en fonction de la 
coloration de Gram et, le tableau 2 renseigne sur la fréquence des bactéries Gram négatives mésophiles et psychrotrophes en fonction de leur comportement sur milieu de Hugh et Leifson glucosé et de leur réponse au test de l'oxydase.

\section{Evolution de la flore bactérienne au cours de la réfrigération}

Nous venons de voir qu'en nombre la flore aérobie mésophile ne varie pas de manière significative $\left(1.10^{4} / \mathrm{ml}\right.$ au stade $J$ et $1,5.10^{4}$ à $\mathrm{J}+4$ ), ce qui n'est pas le cas pour la flore aérobie psychrotrophe.

On peut alors se poser la question de savoir si, au cours de la réfrigération, les espèces bactériennes dominantes sont les mêmes qu'à l'origine : le tableau 3 ci-dessous en donne la réponse.

\subsection{Flore aÉROBIE MÉSOPHILE}

A la lecture du tableau 3, nous observons qu'au cours de $96 \mathrm{~h}$ de réfrigération à $4^{\circ} \mathrm{C}$, la flore mésophile initialement constituée par 82 p. 100 de bactéries Gram positives et par 17,6 p. 100 de bactéries Gram négatives présente une diminution dans la proportion des espèces Gram positives et une augmentation des espèces Gram négatives lesquelles représentent respectivement 56,5 p. 100 et 43 p. 100

En ce qui concerne les espèces bactériennes isolées, les Micrococcaceae qui à l'origine représentaient 71,5 p. 100 de la flore aérobie mésophile ne sont présentes que pour environ 51 p. 100 après $96 \mathrm{~h}$ de réfrigération à $4^{\circ} \mathrm{C}$. Nous identifions Micrococcus avec les espèces M. luteus, M. Varians classées dans les sous-groupes 7 et 5 de Baird Parker $[4,16]$; Staphylococcus avec les espèces $S$. epidermidis (sousgroupe III et II) et $S$. aureus.

Les Bacillaceae identifiées, $B$. licheniformis et $B$. subtilis représentent 10,6 p. 100 de la flore aérobie mésophile initiale et environ 5 p. 100 seulement après une durée de réfrigération de 4 j. Ces deux espèces de Bacillus sont celles qui, le plus souvent, sont identifiées à partir des laits crus réfrigérés étudiés [6, 48, 84, 67].

Parmi la flore bactérienne Gram négative dominante, nous identifions initialement (stade J) 11 p. 100 de Pseudomonas non fluorescents représentés par les espèces $P$. alcaligenes et $P$. pseudomallei, environ 5 p. 100 d'Enterobacteriaceae avec E. coli, E. hafniae, E. agglomerans, S. liquefaciens et Aeromonas hydrophila (1 p. 100).

Au cours des 96 h de réfrigération à $4^{\circ} \mathrm{C}$, nous observons contrairement à ce qui se passe pour la flore Gram positive, une augmentation de la proportion des espèces Gram négatives caractérisées essentiellement par des Pseudomonas fluorescents $[16,70]$ et représentées par $P$. putida et $P$. fluorescens biotype I et $\mathrm{M}$; de plus, comme Buttiaux [14], Millière [50], Pickett [58], Stanier [70], et Véron [83], nous identifions des espèces non pigmentées désignées par le terme «P. fluorescens apigmenté ». 


\section{TABLEAU 3}

Evolution des flores bactériennes aérobies mésophile et psychrotrophe, au cours de $96 \mathrm{~h}$ de réfrigération du lait cru à $4^{\circ} \mathrm{C}$

(résultats donnés en p. 100)

\begin{tabular}{|c|c|c|c|c|}
\hline \multirow[b]{2}{*}{$\begin{array}{l}\text { Genres et } \\
\text { espèces bactériennes }\end{array}$} & \multicolumn{2}{|c|}{$\begin{array}{l}\text { Flore mésophile } \\
\qquad\left(30^{\circ} \mathrm{C}-72 \mathrm{~h}\right)\end{array}$} & \multicolumn{2}{|c|}{$\begin{array}{l}\text { Flore psychrotrophe } \\
\qquad\left(7^{\circ} \mathrm{C}-10 \mathrm{j}\right)\end{array}$} \\
\hline & $\begin{array}{c}\text { Stade } \\
(\mathbf{J})\end{array}$ & $\begin{array}{l}\text { Stade } \\
(J+4)\end{array}$ & $\begin{array}{l}\text { Stade } \\
\text { (J) }\end{array}$ & $\begin{array}{l}\text { Stade } \\
(J+4)\end{array}$ \\
\hline Bactéries Gram + & 82 & 56,5 & 41,4 & 11,6 \\
\hline Micrococcus (sg 5 et 7 ) & 32,5 & 19,3 & 32,6 & 8,6 \\
\hline Sta. aureus & 29 & 16,6 & 0 & 0 \\
\hline Sta. epidermidis (sg III et II) & 10 & 15,3 & 7,4 & 2,4 \\
\hline Bacillus (licheniformis subtilis) & 10,6 & 5,3 & 1,4 & 0,6 \\
\hline Bactéries Gram - & 17,6 & 43 & 58,3 & 88,1 \\
\hline P. pseudomallei & 4,1 & 2,6 & 5,2 & 0,6 \\
\hline P. alcaligenes & 7,1 & 1,3 & 0,7 & 0,6 \\
\hline $\begin{array}{l}P . \text { fluorescents : } P \text {. putida } \\
P . \text { fluorescens }(\mathrm{B} 1 \text { et } \mathrm{BM}) \\
P \text {. fluorescents apigmentés : } \\
P \text {. fluorescens (B1 et BM) }\end{array}$ & $\begin{array}{l}0 \\
0\end{array}$ & $\left.{ }_{12,6\}}^{18,6\}}\right\} 31,2$ & $\left.{ }_{9,6}^{20,7}\right\} 30,3$ & $\left.\begin{array}{l}59,2) \\
14,2\}\end{array}\right\}$ \\
\hline Achromobacter & 0 & 0,6 & 13,3 & 2,4 \\
\hline Aeromonas hydrophila & 1,1 & 0 & 1,4 & 0 \\
\hline $\begin{array}{l}\text { E. coli, Ent. hafniae } \\
\text { Ent. agglo, S. liquefaciens }\end{array}$ & 5,3 & 7,3 & 7,4 & 11,1 \\
\hline
\end{tabular}

\subsection{FLORE AÉROBIE PSYCHROTROPHE}

Les résultats mentionnés dans le tableau 3 nous permettent d'observer que la flore psychrotrophe initialement constituée de 41,4 p. 100 de bactéries Gram positives et de 58,3 p. 100 de bactéries Gram négatives présente, à l'issue de $96 \mathrm{~h}$ de réfrigération à $4^{\circ} \mathrm{C}$, une augmentation considérable de la proportion des espèces Gram néga- 
tives, lesquelles constituent 88,1 p. 100 de la flore psychrotrophe dans ces conditions.

Parmi les espèces bactériennes déterminées, il importe de préciser que $S$. aureus, présent dans les échantillons de lait, n'est pas identifiẻ à $7^{\circ} \mathrm{C}$; ceci étant vraisemblablement lié, comme l'ont montré plusieurs auteurs $[22,31,61]$ aux conditions de développement à basse température, particulièrement défavorables aux espèces mésophiles.

La proportion de 32,6 p. 100 de Micrococcus identifiée dans la flore psychrotrophe initiale (stade J) est caractérisée par l'espèce $M$. varians. L'importance de la proportion de Micrococcus isolé à partir de notre étude comme à partír de celles effectuées par Bockelmann [11], Brandl [12], Kleter [38], Ogawa [53] et Ostenhajmer [56] montre que Micrococcus constitue une partie importante de la flore psychrotrophe des laits paucimicrobiens.

Après $96 \mathrm{~h}$ de réfrigération à $4^{\circ} \mathrm{C}(\mathrm{J}+4)$, la flore psychrotrophe ne renferme que 11,6 p. 100 de bactéries Gram positives représentées par 8,6 p. 100 de Micrococci, 2,4 p. 100 de S. epidermidis et 0,6 p. 100 de $B$. licheniformis.

La variation significative en nombre de la flore aérobie psychrotrophe $\left(5,10^{2} / \mathrm{ml}\right.$ au stade $\mathrm{J}$ et $4,10^{3} / \mathrm{ml}$ à $\left.\mathrm{J}+4\right)$ semble étroitement liée à l'augmentation de proportion des bactéries Gram négatives identifiées qui, de 58,3 p. 100 (stade J) représentent à elles seules, à l'issue de $96 \mathrm{~h}$ de réfrigération à $4^{\circ} \mathrm{C}, 88,1$ p. 100 de la flore psychrotrophe.

Les résultats obtenus à partir de ce lait paucimicrobien nous permettent de déduire que l'action du froid se traduit par une sélection entraînant un profond déséquilibre de la flore bactérienne. Cette sélection favorise ainsi la multiplication des espèces psychrotrophes Gram négatives, initialement peu nombreuses dans le lait et, défavorise les espèces mésophiles Gram positives.

Le genre Pseudomonas identifié représente à lui seul 74,6 p. 100 de la flore psychrotrophe au stade $\mathrm{J}+4$. Les 59,2 p. 100 d'espèces fluorescentes déterminées sont représentées par $P$. putida $(9,2$ p. 100), $P$. fluorescens biotype I (45 p. 100) et $P$. fluorescens biotype M (5 p. 100).

A partir de nos échantillons de lait cru et des résultats obtenus par Juffs [34], Bockelmann [10], Nakae [51], Witter [85] et Thomas $[79,80]$, les Pseudomonas élaborant un pigment vert fluorescent représentent les espèces dominantes de la flore psychrotrophe des laits réfrigérés. Ainsi, nous observons une similitude entre la proportion des Pseudomonas fluorescents identifiés à partir de notre lait paucimicrobien et les résultats compris entre 46 et 50 p. 100 obtenus par Bockelmann [10], Dempster [18] et Nakanishi [52]. D'après certains auteurs, la proportion de ces espèces élaborant un pigment fluorescent peut représenter 50 à 60 p. $100[51,54,63]$ et parfois environ 80 p. 100 [36, 49] de la flore psychrotrophe.

A l'issue de la réfrigération, parmi les 11,1 p. 100 d'Enterobacteriaceae isolées, nous identifions Enterobacter hafniae et Serratia lique- 
faciens. D'une manière analogue, Enterobacter $[3,64,27]$ et Serratia $[74,76,78]$ sont le plus souvent identifiés par les auteurs et représentent en moyenne 4 à 20 p. 100 de la flore psychrotrophe dominante des laits réfrigérés $[9,18,75,77,47,33,52,46,64]$.

La proportion de 2,4 p. 100 concernant Achromobacter sp. identifié dans la flore psychrotrophe à ce stade $(J+4)$ est faible dans nos échantillons, comparativement aux résultats voisins de 11,5 p.100, 28 p. 100,45 p. 100 et 58 p. 100 rapportés respectivement par Kleter [38], Nakae [51], Sekar [65] et Thomas [77].

\section{CONCLUSION}

A l'origine, nous constatons que la flore bactériene de lait paucimicrobien appartient en grande partie au groupe des bactéries Gram positives (71,5 p. 100) de la famille des Micrococcaceae. La réfrigération du lait à $4^{\circ} \mathrm{C}$ pendant $4 \mathrm{j}$ entraîne une modification importante dans la composition de la flore bactérienne originelle. En effet, au cours du stockage du lait à $4^{\circ} \mathrm{C}$, nous voyons diminuer la proportion des espèces mésophiles Gram positives et augmenter de façon significative la flore psychrotrophe Gram négative appartenant pour 73 p. 100 au genre Pseudomonas. Les espèces les plus fréquemment identifiées sont $P$. fluorescens biotypes I et $\mathrm{M}$ et $P$. putida. La proportion de ces bactéries dans le lait réfrigéré dépend de la température et de la durée de réfrigération, du degré de contamination initiale et de la nature des espèces bactériennes à l'origine.

Nous pouvons conclure que dans le cas de laits paucimicrobiens conservés entre $4^{\circ} \mathrm{C}$ et $7^{\circ} \mathrm{C}$ pendant 3 à $4 \mathrm{j}$ et dont la contamination initiale est inférieure ou égale à $10^{4}$ par ml, 16 p. $100[1,60]$ de la flore mésophile est constituée de bactéries psychrotrophes. Par contre, si à l'origine le dénombrement se situe entre $10^{4}$ et $10^{6}$ par $\mathrm{ml}$, les auteurs signalent que cette proportion peut varier de 40 p. 100 [73] à 75 p. 100 [42]. Dans ces conditions, aux températures de réfrigération habituelles, les bactéries psychrotrophes thermolabiles en général, sont capables d'élaborer lipases [1] et protéases [50] suffisamment thermorésistantes qui peuvent contribuer de façon non négligeable à l'altération des laits réfrigérés fortement contaminés [19, 41].

Quoi qu'il en soit, que le lait soit à l'origine paucimicrobien [1] ou fortement contaminé $[36,42,49,54,64]$, les espèces psychrotrophes dominantes identifiées sont les Pseudomonas et plus particulièrement $P$. fluorescens.

\section{Rés u m é}

A l'origine, la flore bactérienne d'un lait cru paucimicrobien est composée de 71,5 p. 100 de Micrococcaceae. Après stockage à $4^{\circ} \mathrm{C}$ pen- 
dant $4 \mathrm{j}$, il s'ensuit une sélection bactérienne qui favorise la prolifération notable des bactéries psychrotrophes Gram négatives initialement présentes en petit nombre dans le lait.

Les Pseudomonas fluorescents représentent quant à eux respectivement 31 p. 100 et 73,4 p. 100 des flores aérobies mésophile et psychrotrophe à l'issue de sa conservation pendant $4 \mathrm{j}$ à $4^{\circ} \mathrm{C}$.

\section{S u m m a r y}

\section{EVOLUTION AND DETERMINATION OF THE BACTERIAL FLORA OF A GRADE A REFRIGERATED RAW MILK DURING STORAGE}

At the beginning, Micrococcaceae constitue 71,5 p. 100 of a grade A milk bacterial flora. Some proliferation of originally few gram negativs psychrotrophic bacteria seems to be induced after storage of milk at $4^{\circ} \mathrm{C}$ during 4 days. Under these conditions, fluorescent Pseudomonas represent 31 p. 100 of the mesophilic bacterial flora and 73,4 p. 100 of the psychrotrophic bacterial flora.

Reçu pour publication en juin 1980.

\section{Bibliographie}

[1] Bloquel (R.) (1979). — Thèse Docteur Spéc. I.N.P.L., Nancy, 534 réf.

[2] Bloquel (R.) et Veillet-Poncet (L.) (1980). - Qualité bactériologique d'un lait cru paucimicrobien réfrigéré à la ferme. Bull. Sci. de l'E.N.S.A.I.A., Nancy, tome XXII (à paraître).

[3] ANDRey (J.) and Frazier (W. C.) (1959) - Psychrophilic in milk held two day in farm milk. J. Dairy Sci., 42 (11), 1781-1784.

[4] BAIRD-PARKeR (A. C.) (1963). - A classification of micrococci and staphylococci based on physiological and biochemical tests. J. Gen. Microbiol., 409-427.

[5] Baumann (P.), Doudoroff (M.) and Stanier (R. Y) (1968). - a) A study of the Moraxella group I genus Moraxella and the Neisseria catarrhalis group. b) A study of the Moraxella group II oxidase-negative species (genus Acinetobacter). J. Bacteriol., 95, 58-73 (a) ; 1520-1541 (b).

[6] Bernhard (E.) (1962). - Aerobe Sporenbildner im Rohmilch. Int. Dairy Congr., vol. C, section VIII, 1, 337-340.

[7] Black (W. A.) and Hodgson (R.) (1971). - Evaluation of three methods using deoxyribonuclease production as a screening test for Serratia marcescens. J. Clin. Path., 24, 313-316.

[8] Blazevic (D. J.) (1976). - Current taxonomy and identification of nonfermentative gram negative bacilli. Human. pathol., 7 (3), 265-275.

[9] Bockelmann (I.) Von (1966). - Gesandkeimzahl und psychrophilic Bakterien tiefgekühlter Milch. Milchwiss., 21 (5), 275-278.

[10] Bockelmann (I.) Von (1970). - Bactéries lipolytiques et psychrotrophes dans le lait gardé au froid. XVIII ${ }^{e}$ Congrès Int. Lait., $1 F, \mathrm{~A} 2-3,109$. 
[11] Bockelmann (I.) Von (1974), - Croissance de la flore bactérienne du lait pendant un stockage prolongé au froid. XVIII e Congrès Int. Lait., $1 F, 440$.

[12] BRANDL (E.) (1968), - Die Mikroflora der Rohmilch und ihre Beeinflussung durch die Pasteurisierung der Milch. D.M. Zeitung, 22 (33), 1109-1114.

[13] Buttiaux (R.) et Gagnon (P.) (1958-1959). - Au sujet de la classification des Pseudomonas et Achromobacter. Ann. I. Pasteur (Lille), vol. X, 121-149.

[14] Buttiaux (R.) (1961). - Pseudomonas non pigmentés et Achromobacter. Ann. Inst. Pasteur, 100, suppl, au $\mathrm{n}^{\circ}$ 6, 43-58.

[15] Buttiaux (R.), Beerens (H.) et Taquet (A.) (1974). - Manuel de techniques bactériologiques. $4^{\mathrm{e}}$ éd., Flammarion (Médecine-Sciences), Paris 6e, $700 \mathrm{p}$.

[16] Cowan (S. T.), Holt (J. G.), Liston (J.), Murray (R. G. E.), Niven (C. F.), RAvin (A. W.) and StanieR (R. Y.) (1974). - Bergey's manual of determinative bacteriology. 8th ed., Buchanan R.E. and Gibbons N.E., co-editors, The Williams and Wilking Company, Baltimore.

[17] de BarJac (H.) et Bonnefor (A.) (1972). - Essai de classification biochimique de 64 Bacillus des groupes II et III représentant 11 espèces différentes. Ann. Inst. Pasteur, 122, 463-473.

[18] DempsteR (J.F.) (1968). - Distribution of psychrophilic micro-organisms in different dairy environments. J. Appl. Bacteriol., 31 (3), 290-301.

[19] Downey (W. K.) and Cogan (J. M.) (1975). - Proceeding of the lipolysis symposium Cork (Ireland). I.D.F., Doc. $\mathrm{n}^{\circ}$ 86, 198 p.

[20] EdWARds (P. R.) and EwING (W. H.) (1972). - Identification of Enterobacteriaceae. 3th ed., 262 p., Burgess Publishing Company, Minneapolis.

[21] EWING (W. H.) and JoHnson (J.G.) (1960). - The differenciations of Aeromonas and 27 cultures from Enterobacteriaceae. Inst. Bull. Bact. Nomencl. Tax., 10, 223-230.

[22] FARRel (J.) and Rose (A. H.) (1967). - Temperature effects on microorganisms. Bact. Rev., 21, 101-120.

[23] Gilardi (G. L.) (1968). - Diagnostic criteria for differentiation of pseudomonas pathogenic for man. Appl. Microbiol., 16 (10), 1494-1502.

[24] Gilard (G. L.) (1969). - Characterization of the oxidase-negative gram $\theta$ coccobacilli (the Achromobacter-Acinetobacter group). Antonie van Leenwenhoeck, 35, 421-429.

[25] GILARdi (G. L.) (1973). - Non fermentative gram-negative bacteria encountered in clinical species. Antonie van Leeuwenhoek, 39 (2), 229-242.

[26] GiLARDI (G. L.) (1976). - Identification of non fermentative gram-negative bacteria. Personal note, 1-8.

[27] Gyllenberg (H. G.) (1968). - Classification des organismes psychrophiles. Bull. Inter. F.I.L., Congrès Brighton, 1968.

[28] Hendrie (M. S.) and SHewan (J. M.) (1966). - The identification of certain Pseudomonas species. In: Identification Methods for microbiologists part A, Gibbs and Skinner Editors, 1-7.

[29] Henriksen (S. D.) (1976). - Moraxella, Neisseria, Branhamella and Acinetobacter. Ann. Rev. Microbiol., 30, 63-83.

[30] Hugh (R.) and Leifson (E.) (1953). - The taxonomic signifiance of fermentation versus oxidative metabolism of carbohydrates by various gram negative bacteria. I. Bacteriol., 66, 24-26.

[31] INGRAHAM (J. L.) (1969), - Factors wich preclude growth of bacteria at low temperature. Cryobiology U.S.A., 6 (3), 188-193. 
[32] International SUbCOMMitTE ON STAPHyLococci AND Micrococci (1965). - Recommandations of subcommittee. Int. Bull. Bact. Nomencl. Taxon., 15, 109.

[33] Jayashankar (S. R.), Dudani (A. T.) and Iya (K. K.) (1966). - Studies on psychrophilic bacteria in milk. XVIIth Congr. Int. Lait., B, 539-545.

[34] JuFfs (H. S.) (1973). - Identification of Pseudomonas sp. isolated from milk produced in South Eastern Queensland. J. Appl. Bacteriol., 36 (4), 585-598.

[35] KING (E. O.) (1975). - The identification of unusual pathogenic gram negative bacteria. Key for identification of aerobes revised, 1975 special bacteriology section, bacteriology division. Center for disease control Atlanta, Georgia, 30333.

[36] Kiuru (K.), Eklund (E.) et al. (1971). - Die proteolitische Aktivität der psychotrophen Mikroorganismen in der Hofbehältermilch. Milchwiss., $26(3), 138-141$.

[37] Kleter (G.) (1974). - Aseptic milking of cows. Neth. Milk. Dairy J,, 28, 212-219.

[38] Kleter (G.) (1974). - The bacterial flora in aseptically drawn milk. Neth. Milk. Dairy J., 28, 220-237.

[39] KNight (B. C. J. G.) and Proom (H.) (1950). - A comparative survey of the nutrition and physiology of mesophilic species in the genus Bacillus. J. gen. Microbiol., 4, 508-538.

[40] Kovacs (N.) (1956). - Identification of Pseudomonas pyocyanea by the oxidase reaction. Nature, 178, 703 .

[41] Kuzdzal-SAvoie (S.) et Auclair (J.E.) (1975). — La lipolyse dans le lait refroidi. Le Lait, tome LV, 548, 530-543.

[42] Lagrange (W.S.) and Nelson (F. E.) (1961). - Bacteriological evaluation of manufacturing-grade bulk-tank milk. J. Dairy Sci., 44 (8), 1440-1445.

[43] Lemille (F.), de Larjac (H.) et Bonnefor (A.) (1969). - Essai sur la classification biochimique de 97 Bacillus du groupe I appartenant à neuf espèces différentes. Ann. Inst. Pasteur, 116, 808-819.

[44] Le Minor (L.) et Ben Hamida (F.) (1962), - Avantage de la recherche de $\beta$-galactosidase sur celle de la fermentation du lactose en milieu complexe dans le diagnostic bactériologique des Enterobacteriaceae. Ann. Inst. Pasteur, 102 (3), 267-277.

[45] Le Minor (L.) (1972). - Le diagnostic de laboratoire des bacilles à gram négatif. Entérobactéries, 226 p., éd. de la Tourelle, Saint-Mandé.

[46] Marth (E. H.) and Frazier (W. C.) (1957). - Bacteriology of milk held at farm bulk cooling tank temperatures. II. Effect of numbers of bacteria in the original milk. I. Effect of time and temperature during storage. J. Milk Fd. Technol., 20 (2), $72-78$; (3), 79-83.

[47] Mc Kenzie (D. A.) (1966). - Bacterial count, coliform and resazurin tests of raw milk. XVII ${ }^{\mathrm{e}}$ Congr. Int. Lait., $B$, 473-480.

[48] Martin (J. H.) and Stahly (D. P.) (1962). - Sporeforming microorganisms in selected milk supplies. Int. Dairy Congr., vol. C, section VIII : 1, 295-304.

[49] Millitere (J. B.), Ben Rachid (S. M.) et Chadli (A.) (1973), - Bactéries psychrotrophes du lait cru. Arch. Inst. Pasteur, Tunis, 50, 189-210.

[50] Millière (J. B.) (1978), - Détermination de la flore bactérienne caséolytique psychrotrophe et mésophile des laits crus réfrigérés et caractérisation du système enzymatique protéolytique des espèces dominantes. Thèse Docteur-Ingénieur, Nancy I, 449 réf. 
[51] NAKAE (T.) (1970), - Characterization and classification of psychrotrophic bacteria in milk by means of temperature-gradient incubation. Milchw., 25 (3), 161-167.

[52] Nakanishi (T.), Nakae (T.) and Rasan (B. S.) (1967). - Psychrotrophic bacteria of raw milk. Tohoku J. Agric. Res., 18 (4), $247-255$ (in Dairy Sci. Abstr., 30 (10), 547, $\mathrm{n}^{\circ}$ 3576).

[53] Ogawa (M.) (1967). - Source of psychrophilic bacterial flora in raw milk. Jap. J. Dairy Sci., 16, 177-184. Abstr. D.S. Absts., 30 (5), 1968.

[54] ORR (M. J.) and Mc LARTY (R. M.) (1964). - Alternate-day collection of bulk milk. Dairy Ind., 29 (3), 169-173.

[55] ORR (M. J.), Mc LARTY (R.) and Baines (S.) (1965). - Further investigations on alternate day collection of bulk milk. Dairy Ind., 30 (4), 278-284.

[56] Ostenhajmer (I.) and Mitic (S.) (1971). - Characteristic types of psychrotropic bacteria in raw milk. Mljekarstvo, 21 (4), $74-79$ (d'après THomas et al., 1973, Dairy Ind., 38, 61-70).

[57] Peny (J.) et Buissière (J.) (1970) Microméthode d'identification des bactéries. II. Identification du genre Staphylococcus. Ann. Inst, Pasteur, 118 (1), 10-18.

[58] Pickett (M. J.) and Pederson (M. M.) (1968). - Screening procedure for partial identification of non fermentative bacilli associated with man. Appl. Microbiol., 16, 1631-1632.

[59] Popoff (M.) and Veron (M.) (1976). - A taxonomie study of the Aeromonas hydrophila and A. punctata. J. gen. Microbiol., 94 (1), 11-22.

[60] Porubiakova (J.) und Prekopp (I.) (1971). - Studium der psychrophilen Mikroflora in der Schafmilch. Milchwiss., 26 (1), 23-26.

[61] Rose (H. A.) and Evison (L. M.) (1965), - Studies on the biochemical basis of the minimum temperatures for growth of certain psychrophilic and mesophilic microorganisms. J. gen. Microbiol., 38, 131-145.

[62] Schubert (R. H. W.) (1967). - The taxonomy and nomenclature of the genus Aeromonas Kluyver and van Niel, 1936. Part I - part II. Int. J. Syst. Bact., 17, 23-27, 273-279.

[63] Schultze (W. D.) and Olson (J.C.) (1957). - The distribution of psychrophilic bacteria in commercial dairy products. J. Dairy Sci., 40 (6), 602, abstract., M 23.

[64] Schultze (W. D.) and Olson (J. C.) (1960). - Studies on psychrophilic bacteria. II. Psychrophilic coliform bacteria in stored commercial dairy products. J. Dairy Sci., 43 (3), 351-357.

[65] Sekar (C. V.) (1947). - Thesis. University of Wales. Anal. D.S.A., 20 (6), 462.

[66] Seleen (W. A.) and StaRK (C. N.) (1943). - Some characteristics of green fluorescent pigment producing bacteria. J. Bacteriol., 46, 491-500.

[67] Shehata (T. E.) and Collins (E. B.) (1971). - Influence of temperature on the growth psychrophilic strains of Bacillus. J. Dairy Sci., 54 (11), 1579-1582.

[68] Shewan (J. M.), Hongkiss (W.) and Liston (J.) (1954). - A method for the rapid differenciation of certain non pathogenic asporogenous bacilli. Nature, 73, 208-209.

[69] Shewan (J. M.), HobBs (G.) and Hodgkiss (W.) (1960). - A determinative scheme for the identification of certain genera of gram negative bacteria with special reference to the Pseudomonadaceae. J. Appl. Bacteriol., 23, 379-390.

[70] Stanier (R. Y.) (1966). - The aerobic pseudomonas: a taxonomic study. J. gen. Microbiol., 43, 159-271. 
[71] Stenzel (W.) and Mannheim (W.) (1963). - On the classification and nomenclature of some non motile and coccoid diplobacteria exibiting the properties of Achromobacteriaceae. Int. Bull. Bact. Nomencl. Taxon., 13 (4), 195-200.

[72] SutTeR (V.L.) (1968). - Identification of Pseudomonas species isolated from human sources. Appl. Microbiol., 16 (10), 1532-1538.

[73] SWARtLing (F.) (1967), - Influence of cold-storage $\left(0-5^{\circ} \mathrm{C}\right)$ of raw milk on certain properties of the milk. Int. Dairy Fed. a Bull., (3), 6-31.

[74] Thomas (S. B.) and Lewis (E.) (1957), - Some biochemical characteristics of freshly isolated strains of the genus Serratia. J. Appl. Bacteriol., 20 (1), 11-16.

[75] Thomas (S. B.), Hobson (P. M.) and BIRD (E. R.) (1959). - Psychrophilic bacteria in milk. XVth Inter. Dairy Congr., 3 (5), 1334-1340.

[76] Thomas (S. B.) and Griffiths (J. M.) (1960 b). - Psychrophilic bacteria in pasteurised milk. Dairy Eng., 77 (12), 438-444.

[77] Thomas (S. B.) and Druce (R. G.) (1963). - Types of psychrophilic bacteria in milk. Dairy Eng., 80 (10), 378-381.

[78] Thomas (S. B.) and Druce (R. G.) (1971). - Psychrotrophic microorganisms in butter. A review, Part. 2. Dairy Ind., 36 (2), 145-150.

[79] Thомаs (S. B.) and Thомаs (B. F.) (1973). - Psychrotrophic bacteria in refrigerated bulk collected raw milk. Dairy Ind., 38 (1), 11-15 ; (2) 61-70.

[80] Thomas (S. B.) and Thomas (B.F.) (1977). - The bacterial content of milking machines and pipelines milking plants. III. Composition of microflora. Dairy Ind., 42 (7), 19-25.

[81] Thornley (M. J.) (1960). - The differentiation of Pseudomonas from other gram-negative bacteria on the basis of arginin metabolism. J. Appl. Bacteriol., 23, 37-52.

[82] Veillet-Poncet (L.) (1973). - La flore bactérienne indologène aéro-anaérobie des laits pasteurisés conditionnés. Cas particulier d'Aeromonas. Thèse Doctorat Sci. Nat., Univ. Nancy. U.E.R. Alimentation et Nutrition A.O., 9322, 103 p., 363 réf.

[83] Veron (M.) (1961). - Pseudomonas pigmentés. Ann. Inst. Pasteur, 100, suppl. au n $\mathrm{n}^{\circ} 6,16-42$.

[84] Watrous (G. H.), Doan (F. J.) and Josephson (D. V.) (1952). - Some bacteriological studies on refrigerated milk and cream. Bull. Pa. Agric. Exp. Sta., 551, in D.S.A., 14 (9), 785.

[85] Witter (L. D.) (1961). - Psychrophilic bacteria. A review. J. Dairy Sci., 44, 983-1015.

[86] Wolf (J.) and BARKer (A. N.) (1968). - The genus Bacillus: Aids to the Identification of its species. From: Identification methods for microbiologist, 93-109. Academic Press, London, New York, 212 p. 